\title{
Cinnamaldehyde Inhibits Inflammation of Human Synoviocyte Cells Through Regulation of Jak/Stat Pathway and Ameliorates Collagen-Induced Arthritis in Rats ${ }^{[\mathbb{S}}$
}

\author{
Wen-Xiang Cheng, ${ }^{1}$ Shan Zhong, ${ }^{1}$ Xiang-Bo Meng, Nian-Ye Zheng, Peng Zhang, \\ Yun Wang, Ling Qin, and Xin-Luan Wang
}

Translational Medicine R\&D Center, Institute of Biomedical and Health Engineering, Shenzhen Institutes of Advanced Technology, Chinese Academy of Sciences, Shenzhen, China (W.-X.C., S.Z., X.-B.M., P.Z., L.Q., X.-L.W.); University of Chinese Academy of Sciences, Beijing, China (W.-X.C., P.Z., X.-L.W.); Musculoskeletal Research Laboratory of Department of Orthopaedics and Traumatology and Innovative Orthopaedic Biomaterial and Drug Translational Research Laboratory of Li Ka Shing Institute of Health, The Chinese University of Hong Kong, Hong Kong SAR, China (N.-Y.Z., L.Q., X.-L.W.); and Center for Research and Technology of Precision Medicine, College of Life Sciences and Oceanography, Shenzhen University, Shenzhen, Guangdong, China (S.Z., Y.W.)

Received September 25, 2019; accepted February 3, 2020

\begin{abstract}
Cinnamaldehyde (Cin), a bioactive cinnamon essential oil from traditional Chinese medicine herb Cinnamomum cassia, has been reported to have multipharmacological activities including anti-inflammation. However, its role and molecular mechanism of anti-inflammatory activity in musculoskeletal tissues remains unclear. Here, we first investigated the effects and molecular mechanisms of Cin in human synoviocyte cells. Then in vivo therapeutic effect of $\mathrm{Cin}$ on collagen-induced arthritis $(\mathrm{CIA})$ also studied. Cell Counting Kit CCK-8 assay was performed to evaluate the cell cytotoxicity. Proinflammatory cytokine expression was evaluated using quantitative polymerase chain reaction and ELISA. Protein expression was measured by western blotting. The in vivo effect of Cin $(75 \mathrm{mg} / \mathrm{kg}$ per day) was evaluated in rats with CIA by gavage administration. Disease progression was assessed by clinical scoring, radiographic, and histologic examinations. Cin significantly inhibited interleukin (IL)-1 $\beta$-induced IL-6, IL-8, and tumor necrosis factor- $\alpha$ release from human synoviocyte cells. The molecular analysis revealed that Cin impaired IL-6-induced activation of Janus kinase 2 (JAK2), signal transducer and activator of transcription 1 (STAT1), and STAT3 signaling pathway by
\end{abstract}

inhibiting the phosphorylation of JAK2, STAT1, and STAT3, without affecting NF- $\kappa \mathrm{B}$ pathway. Cin reduced collageninduced swollen paw volume of arthritic rats. The antiinflammation effects of Cin were associated with decreased severity of arthritis, joint swelling, and reduced bone erosion and destruction. Furthermore, serum IL-6 level was decreased when Cin administered therapeutically to CIA rats. Cin suppresses IL-1 $\beta$-induced inflammation in synoviocytes through the JAK/STAT pathway and alleviated collagen-induced arthritis in rats. These data indicated that Cin might be a potential traditional Chinese medicine-derived, disease-modifying, antirheumatic herbal drug.

\section{SIGNIFICANCE STATEMENT}

In this study, we found that cinnamaldehyde (Cin) suppressed proinflammatory cytokines secretion in rheumatology arthritis synoviocyte cells by Janus kinase/signal transducer and activator of transcription pathway. The in vivo results showed that Cin ameliorated collagen-induced arthritis in rats. These findings indicate that $\mathrm{Cin}$ is a potential traditional Chinese medicine-derived, disease-modifying, antirheumatic herbal drug.
This work was supported by Sino-Swiss collaborative project from Ministry of Science and Technology and the Swiss National Science Foundation (2015DFG32200 and 156362), National Nature Science Foundation of China (81773964), and Shenzhen Science and Technology Research Funding (JCYJ20180302150101316 and 20170502171625936).

${ }^{1}$ W.-X.C. and S.Z. contributed equally to this paper.

https://doi.org/10.1124/jpet.119.262907.

S This article has supplemental material available at jpet.aspetjournals.org.

\section{Introduction}

Inflammation accounts for a wide range of progressive musculoskeletal diseases (Gallo et al., 2017), including osteoarthritis (Geyer and Schonfeld, 2018), rheumatology arthritis (RA) (Jia et al., 2015), and cervical spondylopathy (Kang et al., 1995). It is a complex process regulated by an array of inflammatory mediators and cytokines (Gibon et al., 2017). Cinnamomum cassia Presl is frequently used in traditional Chinese medicine to treat gastritis, blood circulation disturbances,

ABBREVIATIONS: Akt, RAC-alpha serine/threonine-protein kinase; BS, bone surfaces; BV, bone volume; ClA, collagen-induced arthritis; Cin, cinnamaldehyde CMC, Carboxymethyl cellulose; CT, computed tomography; ERK, extracellular signal-regulated kinases; FLS, fibroblast-like synoviocytes; GAPDH, glyceraldehyde-3-phosphate dehydrogenase; H\&E, hematoxylin and eosin; IL, interleukin; JAK, Janus kinase; LPS, lipopolysaccharide; MTX, methotrexate; NF- $\kappa$ B, nuclear factor $\kappa$-light-chain-enhancer of activated B cells; PCR, polymerase chain reaction; RA, rheumatology arthritis; ROI, region of interest; STAT, signal transducer and activator of transcription; TNF- $\alpha$, tumor necrosis factor $\alpha$. 
and inflammatory disease (Gunawardena et al., 2015), such as Jing Shu Ke Li for treatment of cervical spondylopathy.

Cinnamaldehyde (Cin, 3-phenyl-2-propenal), as a major active constituent of Cinnamomum cassia, is widely used as flavoring agents in foodstuffs (Kim et al., 2007), with many pharmacological activities, including anti-inflammation (Huang et al., 2015), suppression of adipocyte differentiation (Huang et al., 2011), and osteoclastgenesis (Tsuji-Naito, 2008). Cin has been known to have anti-inflammatory activity in a variety of cells, such as human renal tubular cells (Huang et al., 2015), monocyte/ macrophage (Chao et al., 2008), and gastrointestinal cells (Li et al., 2016). Furthermore, Cin exhibits inhibitory effects on proinflammatory cytokines in LPS-stimulated macrophages in vitro by suppressing intracellular signaling (Chao et al., 2008; Kim et al., 2010). Liao et al. (2012) found that Cin strongly suppress nitric oxide, tumor necrosis factor (TNF- $\alpha$ ), and prostaglandin E2 productions in LPS-stimulated macrophages. Intraperitoneal injection of Cin with $5 \mathrm{mg} / \mathrm{kg}$ dose to carrageenan-induced mouse paw edema model improved the paw edema by suppressing proinflammatory cytokine expression and promoting the activities of catalase, superoxide dismutase, and glutathione peroxidase in the paw tissue (Liao et al., 2012). Further in vivo studies showed that Cin inhibits the activation of $\mathrm{NF}-\kappa \mathrm{B}$ in aged rat kidney tissues (Kim et al., 2007) and attenuates the advanced glycation end products-induced inflammation via JAK/STAT signaling in human renal tubular cells (Huang et al., 2015). In addition, Cin affects the biologic behavior of human colorectal cancer cells and induces apoptosis via inhibition of the phosphoinositide 3-kinase/Akt signaling pathway (Li et al., 2016). Overall, these findings depicted Cin as a potential therapeutic agent in inflammatory diseases. However, the possible anti-inflammatory and protective mechanisms of Cin on musculoskeletal cells are not fully understood.

Fibroblast-like synoviocytes (FLS) are located in the knee joint and responsible for most of the musculoskeletal diseases (Rahmati et al., 2016) by releasing large amounts of proinflammatory mediators, including interleukin (IL)-6, IL-8, and TNF- $\alpha$ (Sluzalska et al., 2017). IL-1 $\beta$ is one of the key cytokines stimulating FLS to produce IL-6 and IL-8; thus, many researchers use IL- $1 \beta$-induced fibroblast-like synoviocytes for inflammation experiments (Sluzalska et al., 2017).

In this study, we focused on investigating the effects of Cin on IL- $\beta$-induced inflammatory cytokine production in human MH7A cells derived from RA-FLS (Jia et al., 2015) and joint destruction in collagen-induced arthritis (CIA) animal model and its underlying mechanisms. Our data showed that Cin inhibited IL-1 $\beta$-induced inflammatory cytokine production in MH7A cells mainly via the suppression of JAK/STAT pathways. We also demonstrated that Cin attenuated the severity of arthritis in rats with CIA. These results indicate that Cin may be a potential traditional Chinese medicine-derived, disease-modifying, antirheumatic drug.

\section{Materials and Methods}

Cell Culture. Human rheumatoid fibroblast-like synoviocyte line MH7A were provided by Riken cell bank (Tsukuba, Japan). Cells were incubated in RPMI-1640 medium (Hyclone) with 10\% FBS (Gibco, GrandIsland) and penicillin/streptomycin (1:100; Hyclone) at $37^{\circ} \mathrm{C}$ and $5 \% \mathrm{CO}_{2}$. Primary synovial cells of patients with rheumatoid arthritis were from Peking University Shenzhen Hospital. The cells were incubated in Dulbecco's modified eagle's medium (Hyclone) with $10 \% \mathrm{FBS}$ (Gibco) and penicillin/streptomycin (1:100; Hyclone) at $37^{\circ} \mathrm{C}$ and $5 \% \mathrm{CO}_{2}$. This study was approved by the Medical Ethical Committee of Peking University Shenzhen Hospital and was performed according to the recommendations of the Declaration of Helsinki.

Cell Viability Assay (CCK-8 Assay). MH7A cells were seeded into 96-well culture plates at $5 \times 10^{3}$ cells/well; after 24-hour incubation, cells were treated with or without cinnamaldehyde (Cin, the compound provided by Jing Fang [Anhui] pharmaceutical Limited Company, SinoPharm Group, the purity is 97.96\%) at the concentrations of $20,40,60,80$, and $100 \mathrm{nM}$ for 24 hours. Then, CCK- 8 (Dojindo, Japan) was added and incubated at $37^{\circ} \mathrm{C}$ for 2 hours. The absorbance of CCK- 8 was detected at $450 \mathrm{~nm}$ by a microplate reader (PerkinElmer).

Quantitative Real-Time Polymerase Chain Reaction. MH7A cells were pretreated or untreated with various concentrations of Cin for 2 hours and then incubated for another 6 hours with or without $20 \mathrm{ng} / \mathrm{ml}$ of IL-1 $\beta$ (Peprotech). Total RNAs were isolated using the commercial total RNA miniprep kit (Axygen), according to the manufacturer's instructions. Each sample was reverse transcribed using the cDNA synthesis kit (TaKaRa, China) according to the manufacturer's protocol. The primer sequences were used for realtime PCR as displayed in Table 1. Real-time PCR analysis was performed using SYBR Green PCR Premix Ex Taq II reagents (TaKaRa) on a Light Cycler 480 II real-time system (Roche). The GAPDH was served as house-keeping gene for normalization.

ELISA. MH7A cells and primary synovial cells were seeded at $5 \times$ $10^{6}$ cells/well into six-well plates; after 24 hours, MH7A cells were pretreated with various concentrations of Cin or not for 2 hours and then stimulated with or without IL- $1 \beta(20 \mathrm{ng} / \mathrm{ml})$ for another 24 hours. Another group was stimulated with IL- $1 \beta(20 \mathrm{ng} / \mathrm{ml})$ for 2 hours then treated by Cin for 24 hours. The culture medium was collected, and the concentrations of key cytokines were determined by ELISA with a commercial kit (eBioscience Technology Shenzhen, China), according to the manufacturer's instructions.

Western Blot. MH7A cells were pretreated with Cin, Jak 2 inhibitor (Santa Cruz), or were not pretreated for 2 hours, then stimulated with IL-1 $\beta(20 \mathrm{ng} / \mathrm{ml})$ for 1 hour, and then cellular proteins were assayed by western blot analysis. Total proteins in the cell were lysed by lysis buffer (radio-immunoprecipitation assay; Thermo), and protein concentration was measured by BCA kit (Thermo). $50 \mu \mathrm{g}$ of protein from each sample was separated by $10 \%$ SDS-PAGE and transferred to a polyvinylidene fluoride membrane (Millipore Co.). After blocking with 5\% bovine serum albumin in Tris-buffered saline/ Tween20, the membrane was incubated with specific antibody overnight at $4^{\circ} \mathrm{C}$, followed by incubation with the secondary antibody. The band density was quantified by Image Laboratory (Bio-Rad). Primary antibodies for phospho-JAK2 (Tyr1007/1008), phospho-STAT1 (Tyr701), phospho-STAT3 (Tyr705), phospho-Akt (Thr308), phosphoERK1/2 (Thr202/Tyr204), JAK2, STAT1, STAT3, Akt, ERK1/2, and GAPDH were purchased from Cell Signaling Technology (Beverly), and the antibodies for phospho-NF- $\kappa$ B-p65 (Ser468) and NF- $\kappa$ B-p65 were purchased from Signalway Antibody (College Park, Maryland). The corresponding densitometry analysis was performed using Image Laboratory (Bio-Rad) by calculating the average optical density in each field. The ratio of phosphorylated protein to total protein was qualified for comparison between different groups.

Determination of the Effect of Cin on CIA. CIA was induced in Lewis female rats ( 8 weeks old) by bovine type II collagen (Chondrex, Redmond, WA) in acetic acid $(2 \mathrm{mg} / \mathrm{ml})$, which was emulsified in an equal volume of Incomplete Freund's Adjuvant. Inject $0.2 \mathrm{ml}(200 \mathrm{mg}$ collagen/rat) of the emulsion subcutaneously at the base of the tail. A booster injection has been given on day 7 after the initial immunization with $0.1 \mathrm{ml}$ of the emulsion subcutaneously in the tail (Brand et al., 2007). When swollen joints appeared at day 14 after the first injection, rats (six per each group) were given gavage administration of either Cin (75 mg/kg per day, CIA-Cin) (Weng et al., 2019), 
TABLE 1

Primer sequences for real-time PCR

\begin{tabular}{|c|c|c|}
\hline Gene & Forward Primer $\left(5^{\prime} \rightarrow 3^{\prime}\right)$ & Reverse Primer $\left(5^{\prime} \rightarrow 3^{\prime}\right)$ \\
\hline IL-6 & CCTGACCCAACCACAAATGC & ATCTGAGGTGCCCATGCTAC \\
\hline IL-8 & GGTGCAGTTTTGCCAAGGAG & TTCCTTGGGGTCCAGACAGA \\
\hline TNF- $\alpha$ & CCCCAGGGACCTCTCTCTAATC & GGTTTGCTACAACATGGGCTACA \\
\hline GAPDH & GGAGTCCACTGGCGTCTT & AGGCTGTTGTCATACTTCTCAT \\
\hline
\end{tabular}

methotrexate (MTX, $0.5 \mathrm{mg} / \mathrm{kg}$ per 3 days, CIA-MTX) as positive drug or sodium carboxymethylcellulose (CMC-Na, CIA-CMC-Na) as negative control for 21 days. Another six rats were used as the normal control group. The animals were housed under special pathogen-free conditions at the animal facility. The animal experiments were approved by the Animal Ethics Committee of Chinese Academy of Sciences Shenzhen Institute of Advanced Technology (SIAT-IRB150303-YGS-CWX-A0114).

Paw Volume Assessment. The hind paw volume was measured every week from the first immunization. Place a beaker containing a $10 \%$ soap solution of a known density on a top-loading balance and tare the balance to zero. Light anesthesia may facilitate this process. Immerse the limb in the solution to the level of the anatomic hair line. After retraction of the paw, the container is weighed, subtracted from the start weight and corrected for fluid density.

Micro-Computed Tomography Assessment of Arthritis. The right paws and ankle joints were scanned and reconstructed into a three-dimensional structure by the SKYSCAN 1176 micro-CT (Bruker, Belgium). The settings recommended by the manufacturer with exposure of $60 \mathrm{kV}$ and $400 \mu \mathrm{A}$ was used. Bone volume (BV) and bone surfaces (BS) of the tarsal bones were then computed to evaluate the structural changes. The region of interest (ROI) included the region between the metatarsal and tarsal bones. To evaluate the surface topology of the periarticular bone (indicating focal erosion on the bone surfaces) the ratio BS:BV was used (Antill et al., 2016; Zheng et al., 2018).

Measurement of Serum Cytokine Production in Rats. Rat serum was obtained by centrifugation of blood in serum separator Microtainer tubes after cardiac puncture. The levels of cytokines and chemokines were measured by multiplex immunoassay using MILLIPLEX MAP Rat Cytokine/Chemokine magnetic panel as per manufacturer's instructions (Millipore). We analyzed protein levels of IL- $1 \beta$. Species cross reactivity was evaluated by the manufacturer (http:// www.abacus-als.com/media/Milliplex_2014.pdf).

Histopathology Assessment. After 21 days of treatment, the hind limbs were removed and fixed with $10 \%$ formalin, decalcified for 6 weeks in $10 \%$ EDTA, dehydrated and embedded in paraffin. The sections were stained with hematoxylin and eosin (H\&E) for light microscopy (Chow et al., 2018). The sections were scored for changes in synovial proliferation, inflammation, cartilage damage, and bone erosion using the following scoring system: 0 , normal; 1 , mild; 3 , moderate; and 4, severe (Hayer et al., 2016).

Statistical Analysis. Data were presented as mean \pm S.D. The in vitro data were from at least three independent experiments. The sample size was six per group for in vivo evaluation. Statistical analysis was performed using GraphPad Prism 7.0 (GraphPad Software Inc., San Diego, CA). The significance of differences between groups was determined using Student's unpaired $t$ test. One-way ANOVA followed by Fisher's post hoc was used to evaluate differences between experimental groups. A value of $P<0.05$ was considered to be statistically significant.

\section{Results}

Cin Effects on Cell Viability. To test whether Cin (Fig. 1A) exerted anti-inflammation effects below a cytotoxic threshold, we performed CCK-8 assay to detect the influence of Cin on MH7A cell growth. MH7A cells were cultured in various concentrations ranking from 0 to $100 \mathrm{nM}$ of Cin for 24 hours (Fig. 1B). No cytotoxic effects were found below the $100 \mathrm{nM}$, a dose that caused an $8.74 \%$ reduction in viability $(P<0.01)$. We therefore selected 40,60 , and $80 \mathrm{nM}$ dosages for Cin in subsequent experiments testing its potential therapeutic utility against RA.

Cin Treatment Causes IL-6, IL-8, and TNF- $\alpha$ Level Decreased in IL-1 $\beta$-Stimulated MH7A Cells and Primary Synovial Cells. Using the in vitro IL- $1 \beta$-stimulated MH7A cell and primary synovial cells of inflammation, we next examined whether Cin influenced the accumulation of proinflammatory cytokines IL-6, IL-8, and TNF- $\alpha$. MH7A cells were pretreated with Cin or not for 2 hours and then stimulated with IL- $1 \beta(20 \mathrm{ng} / \mathrm{ml})$. After 6 hours, the mRNA levels of IL- 6 , IL- 8 , and TNF- $\alpha$ were measured by quantitative PCR. The results showed that IL- $1 \beta$ significantly upregulated IL-6, IL-8, and TNF- $\alpha$ gene expressions to $181.8,1167$, and 10.62 folds, respectively $(P<0.001$ for all). Cin at 40, 60, and $80 \mathrm{nM}$ dose-dependently and significantly inhibited the gene expression of IL-6 to $58.08 \%, 46.87 \%$, and $31.91 \%$, respectively $(P<0.001$ for all), whereas Cin at 60 and $80 \mathrm{nM}$ dose-dependently and significantly inhibited the gene expression of IL-8 to $78.28 \%$ and $66.3 \%$, respectively ( $P<0.001$ for both). Furthermore, Cin at 40,60 , and $80 \mathrm{nM}$ dose-dependently and significantly inhibited the gene expression of TNF- $\alpha$ to $76.38 \%, 64.14 \%$, and $53.38 \%$, respectively $(P<0.001$ for all, Fig. 2A).

After the Cin treatment, whether stimulated by IL- $1 \beta$ previously and after, the protein levels of IL-6, IL-8, and TNF- $\alpha$ were measured in the culture medium by ELISA in MH7A (Fig. 2, B and C). IL- $1 \beta$ significantly stimulated the releases of IL-6, IL-8, and TNF- $\alpha$ protein to $88.58,180.4$, and 10.3 folds, respectively $(P<0.001$ for all $)$. Cin at 40,60 , and 80 $\mathrm{nM}$ dose-dependently and significantly inhibited the release of IL-6 to $81.42 \%, 63.2 \%$, and $57.41 \%$, respectively $(P<0.001$ for all). Cin at 40 and $80 \mathrm{nM}$ significantly inhibited the release of IL-8 to $89.4 \%(P<0.05)$ and $78.93 \%(P<0.001)$. Cin at 60 and $80 \mathrm{nM}$ dose-dependently and significantly inhibited the release of TNF- $\alpha$ to $60.19 \%$ and $51.22 \%$, respectively $(P<0.001$ for both). Cin also suppressed the level of proinflammatory factors in primary synovial cells, which is consistent with the results of MH7A cell lines, significantly inhibited the release of IL- 6 and IL-8, and the expression of TNF- $\alpha$ was too low to be detected (Supplemental Fig. 1).

Cin Suppresses JAK/STAT Signaling in IL-1 $\beta$-Induced MH7A Cells. The JAK/STAT cascade is involved in the regulation of numerous developmental and homeostatic processes, including inflammatory responses (Ahmad et al., 2015). To further characterize the role of JAK/STAT signaling in antiinflammation of Cin treatment, we detected the activation degrees of signal protein. MH7A cells were pretreated with Cin 

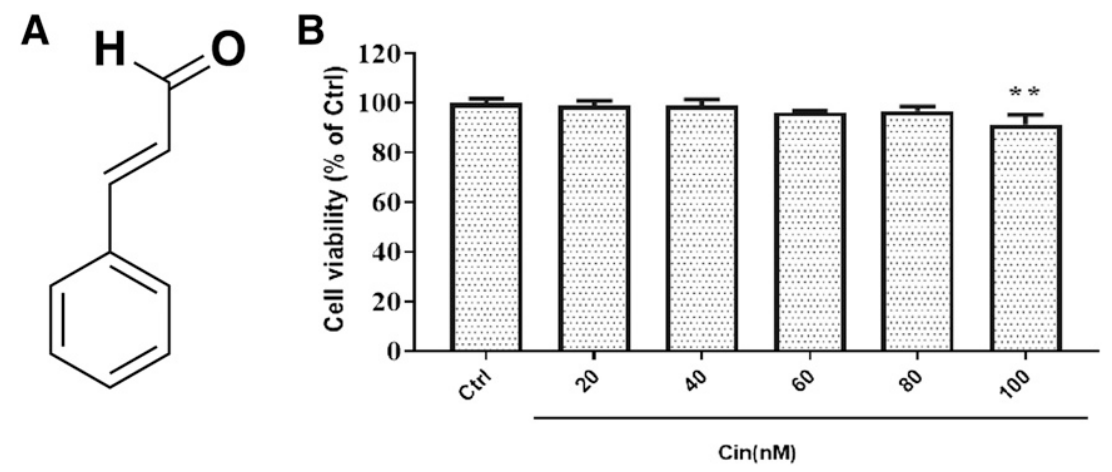

Fig. 1. Effect of Cin on cell viability of MH7A cells by CCK-8. (A) The chemical structure of Cin. (B) CCK-8 results showed that Cin did not have toxic effects at concentrations lower than $100 \mathrm{nM}$. $* P<0.01$ compared with the control group (Ctrl).

or not for 2 hours, and then stimulated with IL-1 $\beta(20 \mathrm{ng} / \mathrm{ml})$ for 15,30 , or 60 minutes, JAK/STAT pathway was determined by western blot analysis.

Cin decreased the ratio of p-JAK2:JAK2 in a dosedependent manner (Fig. 3A1), and $80 \mathrm{nM}$ of Cin significantly decreased the ratio to $52.3 \%$ compared with the IL- $1 \beta$ stimulated only group $(P<0.01)$ (Fig. 3A2). The ratio of p-STAT1:STAT1 and p-STAT3:STAT3 increased by 2.5 - and 2.0 -fold at 60 minutes after IL- $1 \beta$ stimulation, respectively
(Fig. 3B), and Cin at $80 \mathrm{nM}$ dramatically decreased the ratio of p-STAT1:STAT1 and p-STAT3:STAT3 to $42.3 \%$ and $49.5 \%$ compared with the IL- $1 \beta$ stimulated only group $(P<$ 0.01 for both) (Fig. 3B). Compared the effects of $80 \mathrm{nM}$ of Cin and $20 \mu \mathrm{M}$ of Jak 2 inhibitor, at 60 minutes after IL-1 $\beta$ stimulation, $80 \mathrm{nM}$ of Cin could decrease the expression of p-JAK2, p-STAT1, and p-STAT3, whereas Jak 2 inhibitor decreased the expression of p-STAT1 and p-STAT3 but not p-JAK2 (Fig. 3C).
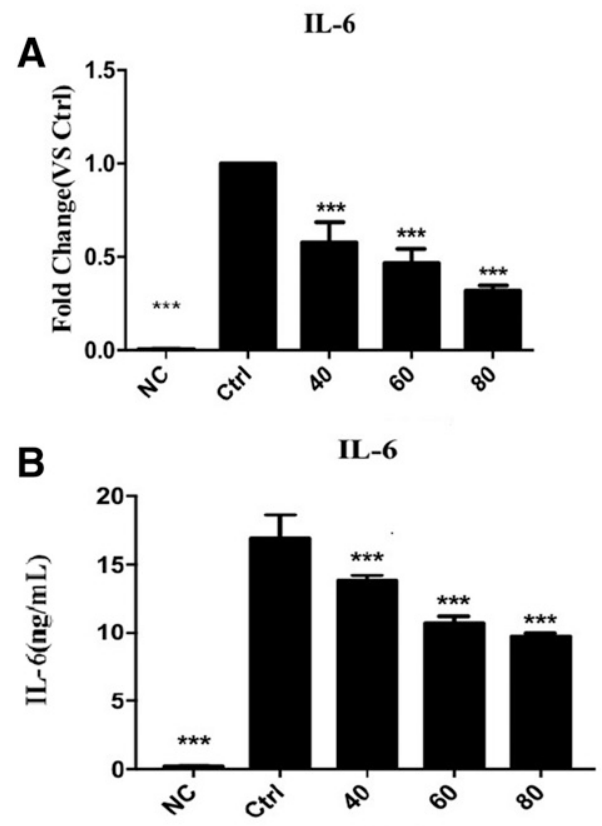

C

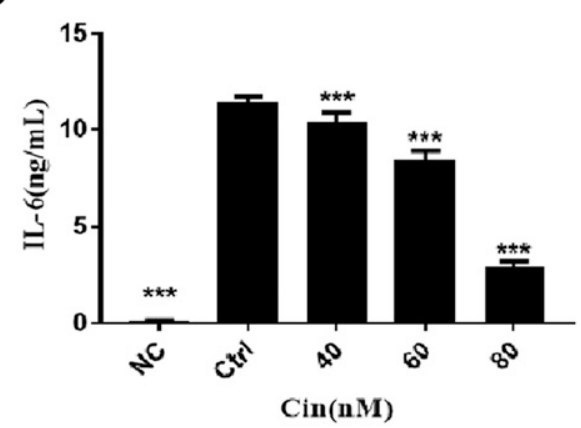

IL-8
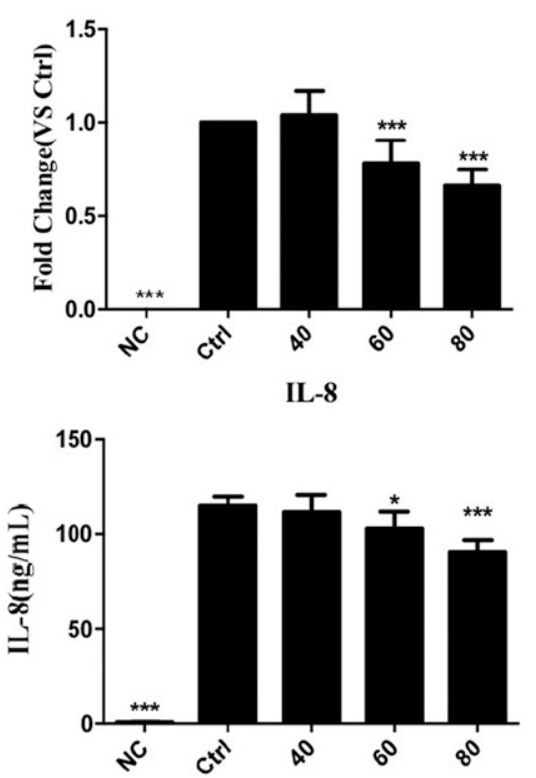

IL-8

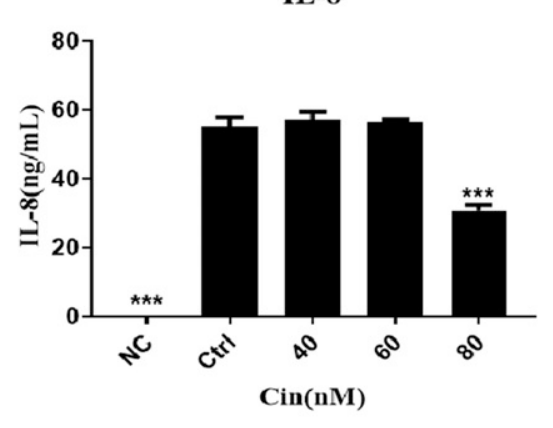

TNF- $\alpha$
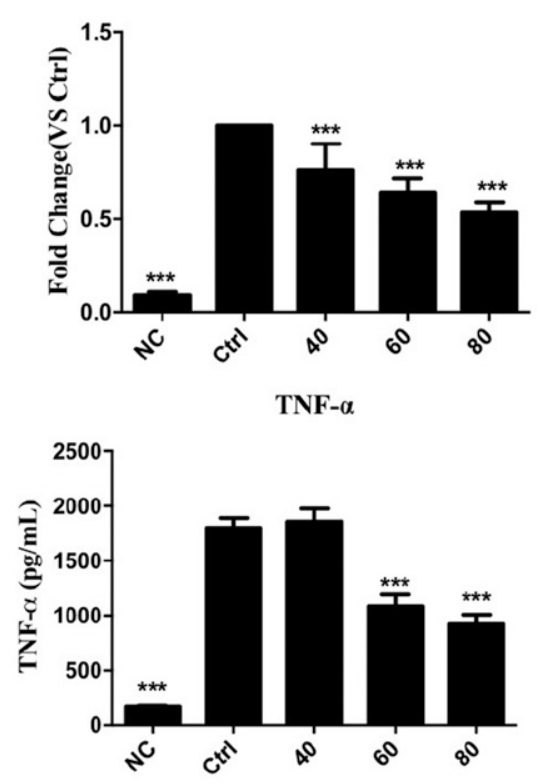

TNF- $\alpha$

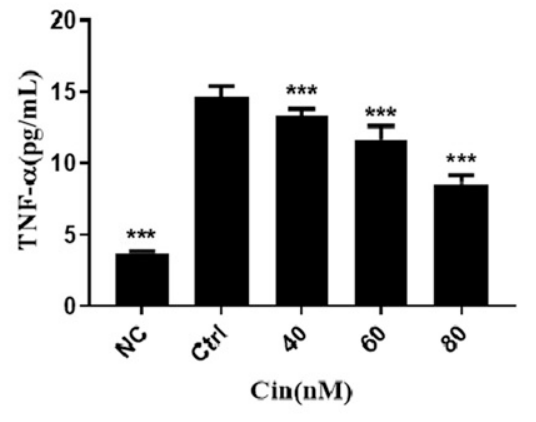

Fig. 2. Anti-inflammation effects of Cin in MH7A. Real-time PCR (A) and ELISA. The result demonstrated that Cin dose-dependently inhibited the production of IL-6, IL-8, and INF- $\alpha$ in IL-1 $\beta$-stimulated MH7A cells. (B) Group for pretreated with Cin first, then stimulated by IL-1 $\beta$. (C) Group for stimulated by IL- $1 \beta$ first, then treated with Cin. ${ }^{*} P<0.05$; $* * *<0.001$ compared with the control group $(\mathrm{Ctrl}$, IL-1 $\beta$ treated alone). 
Cin Promotes Phosphorylation of Akt but Slightly Decreases Phosphorylation of NF- $\kappa$ B in IL-1 $\beta$-Induced MH7A Cells. Inhibition of NF- $\kappa \mathrm{B}$ transcriptional activity is an important way for anti-inflammation in osteoarthritis and RA. Akt and ERK signaling pathways could be the main upstream of NF- $\kappa$ B signaling cascades (Dan et al., 2008; Sun et al., 2017). We observed that the NF- $\kappa \mathrm{B}$ transcriptional activity was affected by $\mathrm{Cin}$ in MH7A cells. MH7A cells were pretreated with or without Cin for 2 hours, followed by stimulation with IL-1 $\beta(20 \mathrm{ng} / \mathrm{ml})$ for 15,30 , or 60 minutes. Cin at 60 and $80 \mathrm{nM}$ significantly increased the ratio of p-AKT: AKT compared with the IL-1 $\beta$ stimulated only group $(P<0.01$ for both) (Fig. 4A). The ratio of p-AKT:AKT was the highest at 15 minutes after IL- $1 \beta$ stimulation, and Cin at $80 \mathrm{nM}$ significantly increased the ratio of p-AKT:AKT at each time point (Fig. 4B). The total protein of ERK1/2 decreased with IL-1 $\beta$ stimulation, and Cin had no effect on the ratio of p-ERK1:2: ERK1/2 (Fig. 4). The ratio of p-NF- $\kappa \mathrm{B}-\mathrm{p} 65: \mathrm{NF}-\kappa \mathrm{B}-\mathrm{p} 65$ did not change obviously with IL- $1 \beta$ stimulation, but $80 \mathrm{nM}$ of Cin could slightly decrease the ratio of p-NF- $\kappa$ B-p65:NF- $\kappa$ B-p65 $(P>0.05)$.

Cin Administration Attenuates the Arthritis Severity in CIA Rats. We next evaluated the in vivo effects of Cin on the synovial inflammation of CIA rats. Compared with the negative control group, a slight body weight loss was noted in the CIA model rats $(P>0.05)$ (Fig. 5A). As shown in Fig. 5, from day 14 onwards, there was a continuous increase of paws and ankle volume. Administration with Cin caused a significant reduction in paw volume (30\%) (Fig. 5B). In agreement with the inhibition of paw volume, treatment with Cin had a marked inhibitory effect on the systemic expression of proinflamatory cytokine IL-1 $\beta$ (Fig. 5C). However, the expression of IL- 6 and TNF $\alpha$ was too low to be detected in serum (unpublished data).
The effects of all treatments on bone erosion in the periarticular bone was analyzed via micro-CT evaluation of the periarticular bone in ankle joints and the distal end of left tibiae with a quantitative histomorphometric imaging method. Micro-CT assessment of the ankle joints of the CIA control group revealed that bone erosions usually involved all small tarsal bones. Micro-CT three-dimensional images showed that the articular surfaces of tarsal bones were very irregular, and the talocalcaneal joint space was frequently obliterated (Fig. 5D). Qualitative data showed that in the Cin group, the surface area of the tarsal bone decreased and BS: BV decreased significantly $(P<0.05)$. The results in the MTX group were similar to that in the Cin group (Fig. 5, E and F).

We also conducted histologic examinations to evaluate the joint pathology in rats. H\&E staining showed that a large number of inflammatory cells infiltrated and bone destruction in localized areas of the CIA control group (Fig. 6A; Supplemental Fig. 2). As expected, Cin-treated rats showed a significant reduction of pathologic disease severity as supported by lower synovial proliferation (Fig. 6B), inflammation (Fig. 6C), cartilage damage (Fig. 6D), and bone surface erosion (Fig. 6E) than those in the CIA group. Similarly, MTX also alleviated the histopathological arthritic damage in the CIA joints (Fig. 6, B-E). These data suggested that Cin could alleviate inflammation and prevent from bone destruction.

\section{Discussion}

In the present study, the anti-inflammatory effect of Cin were explored in a rat model of collagen-induced arthritis and in IL- $1 \beta$ treated human rheumatoid fibroblast-like synoviocytes line MH7A, respectively. Cin exhibited the inhibitory potential against the proinflammatory cytokines via the
A1

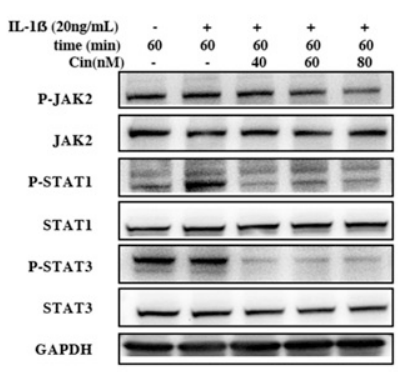

A2

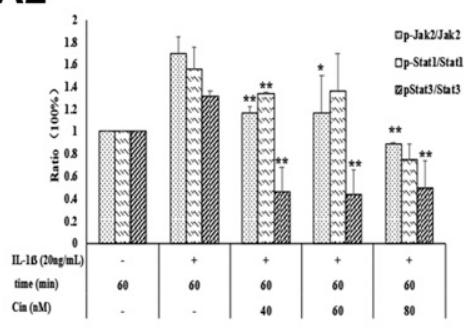

B1

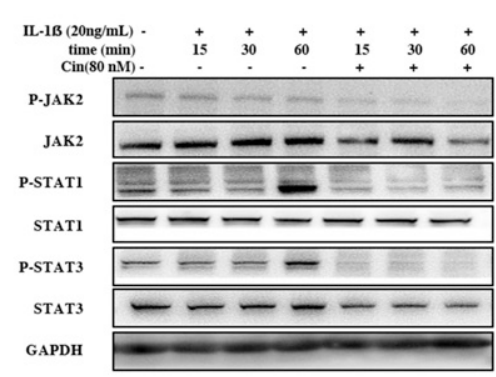

B2

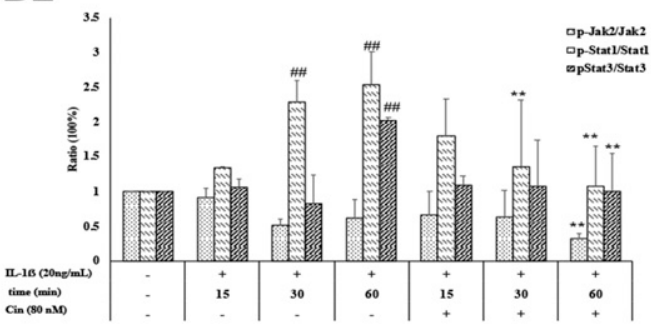

C1

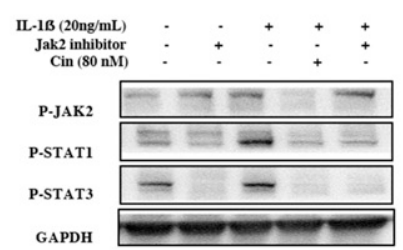

C2

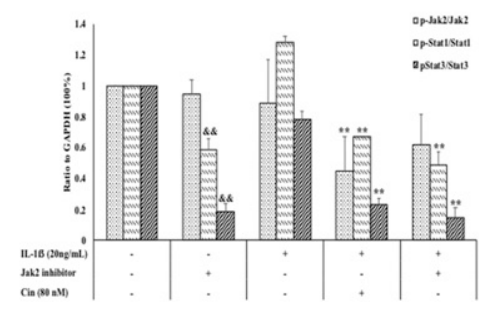

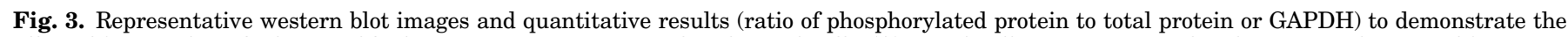

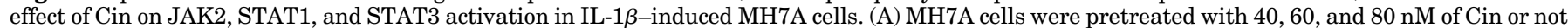

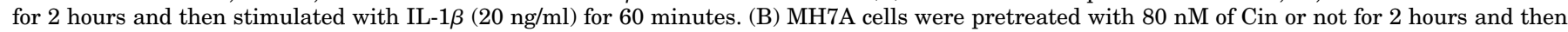

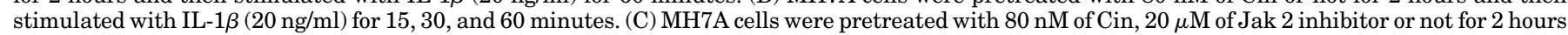

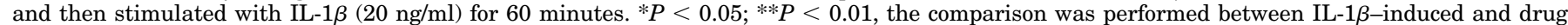

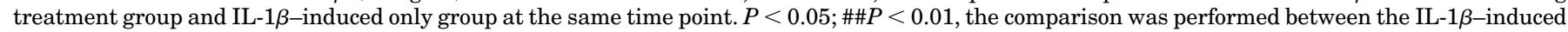

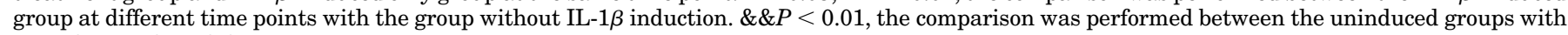
or without Jak2 inhibitor treatment. 


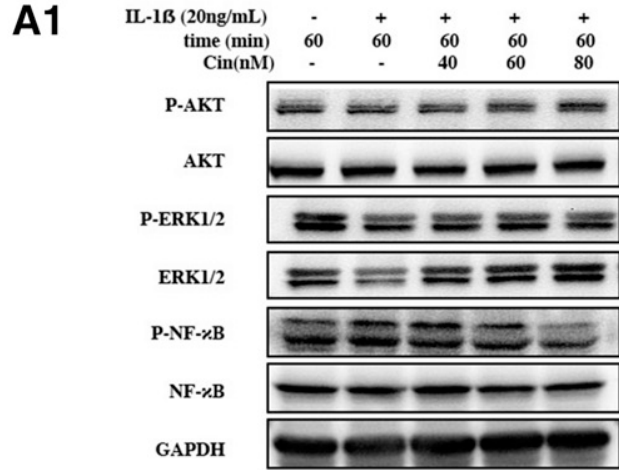

A2

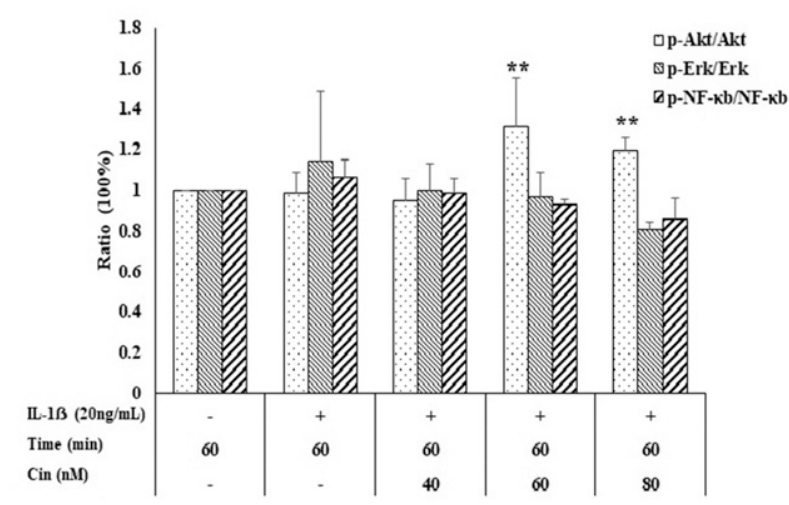

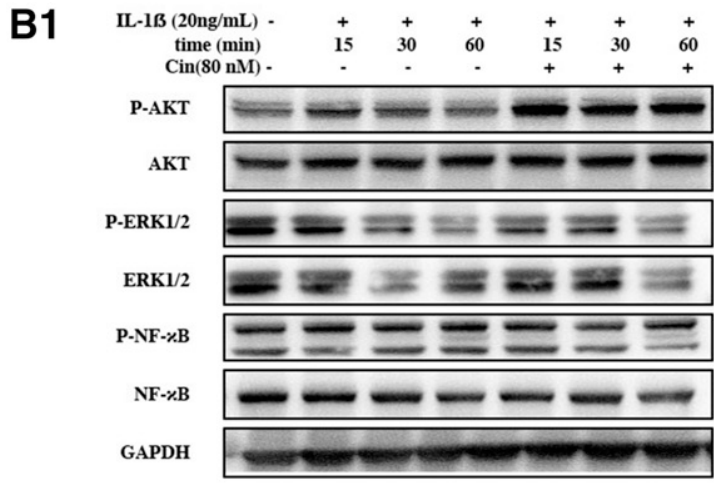

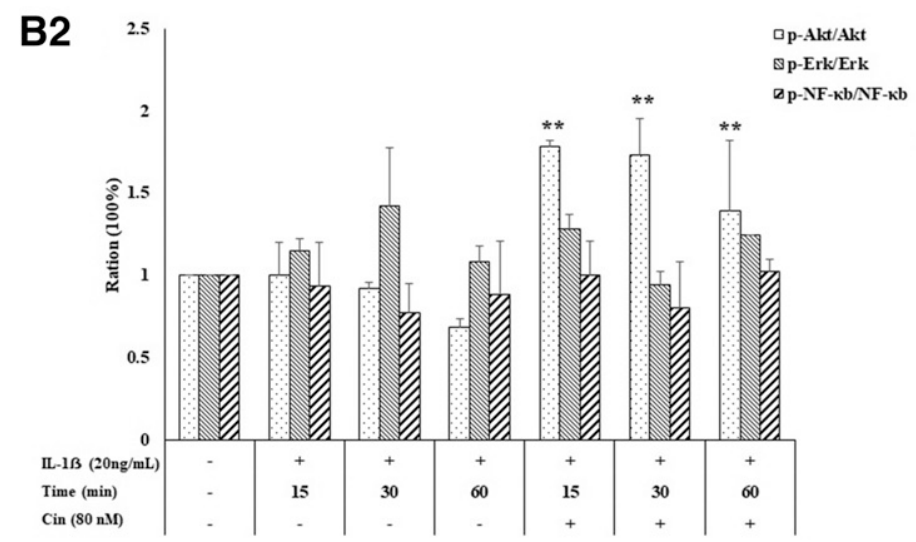

Fig. 4. Representative western blot images and quantitative results (ratio of phosphorylated protein to total protein) demonstrated the effect of Cin on AKT, ERK, and NF- $\kappa$ B activation in IL-1 $\beta$-induced MH7A cells. (A) MH7A cells were pretreated with 40,60 , and $80 \mathrm{nM}$ of Cin or not for 2 hours and then stimulated with IL-1 $\beta(20 \mathrm{ng} / \mathrm{ml})$ for 60 minutes. (B) MH7A cells were pretreated with $80 \mathrm{nM}$ of Cin or not for 2 hours and then stimulated with IL-1 $\beta$ $(20 \mathrm{ng} / \mathrm{ml})$ for 15,30 , and 60 minutes. $P<0.05$; $* * P<0.01$, the comparison was performed between IL- $1 \beta$-induced and Cin treatment group and IL$1 \beta$-induced only group at the same time point.Representative western blot images and quantitative results (ratio of phosphorylated protein to total protein) demonstrated the effect of Cin on AKT, ERK, and NF-кB activation in IL-1 $\beta$-induced MH7A cells. (A1) MH7A cells were pretreated with 40, 60, and $80 \mathrm{nM}$ of Cin or not for 2 hours and then stimulated with IL-1 $(20 \mathrm{ng} / \mathrm{ml})$ for 60 minutes. (A2) MH7A cells were pretreated with $80 \mathrm{nM}$ of Cin or not for 2 hours and then stimulated with IL-1 $(20 \mathrm{ng} / \mathrm{ml})$ for 15,30 , and 60 minutes. (B1 \& B2) Corresponding densitometric analysis of A1 \& A2. *P $<.05$ compared with the control group.The ${ }^{*} \mathrm{P}<0.05$; ${ }^{* *} \mathrm{P}<0.01$, the comparison was performed between IL-1 $\beta$-induced and Cin treatment group and IL$1 \beta$-induced only group at the same time point.

suppression of JAK/STAT signaling pathway in in vitro assay. Moreover, systemic administration of Cin exerted an antiarthritic effect as evidenced by a decrease in paw swelling and significant reduction in histologic changes in the articular joints of rats. We also demonstrated that systemic induction of Cin therapy significantly reduced the serum levels of proinflammatory cytokine IL- $1 \beta$.

Cytokine concentrations correlated with clinical symptoms, inflammatory indicators, disease activities, and serum biomarkers (Yuan et al., 2018). Thus, the proinflammatory productions of TNF- $\alpha$, IL- 6 , and IL- 8 were measured after Cin treatment to assess the anti-inflammatory outcome. The releases of cytokines, especially TNF- $\alpha$, IL- $1 \beta$, IL- 6 , and IL- 8 , were critically involved in the inflammation process (Swärd et al., 2012; Cassuto et al., 2018). IL-1 $\beta$ possesses a broad spectrum of proinflammatory properties and is usually used for including inflammation in vitro (Castejónet al., 2017; Feng et al., 2017). It could induce the synthesis of proinflammatory cytokines (such as TNF- $\alpha$ and IL-6), chemokines (such as IL8), and activated macrophages (Castejónet al., 2017; Feng et al., 2017). Previous studies showed that Cin $(80 \mu \mathrm{M})$ inhibited the secretion of IL- $1 \beta$ and TNF- $\alpha$ within LPS or lipoteichoic acid stimulated murine J774A.1 macrophages (Chao et al., 2008). In our study, Cin at the concentrations of
60 and $80 \mathrm{nM}$ significantly suppressed IL- $1 \beta$-activated inflammatory cytokine production levels in human synoviocyte cell line MH7A. It also inhibited IL-6 and IL-8 cytokines levels in primary synovial cells. The $\mathrm{TNF} \alpha$ was not detectable in this primary cell, which may be due to the different cell resources as well as the cells state and the limited primary sample size (Guo et al., 2016). Consistent with the cell ELISA results, Cin led to a decrease in serum cytokine levels of IL- $1 \beta$ in CIA rats, suggesting that Cin might provide beneficial effects by specific down-regulating the synthesis of IL- $1 \beta$.

The JAK/STAT cascade has been shown to be involved in the regulation of numerous developmental and homeostatic processes, including inflammatory responses (Ahmad et al., 2015). IL-1 $\beta$ binding to their receptors can induce the phosphorylation of receptor-associated JAK in chondrocytes, which in turn transduces the intracellular signal by further phosphorylating and activating STATs (Lim and Kim, 2011). The STATs are activated by phosphorylation at the tyrosine residue, and then dimerized, translocated to the nucleus and bind DNA, initiating the transcription of target genes (Adach et al., 2009; Qi and Yang, 2014).

Available literature reported that Cin attenuated advanced glycation end products-induced the JAK/STAT signaling in human renal tubular cells (Huang et al., 2015). We observed 
A

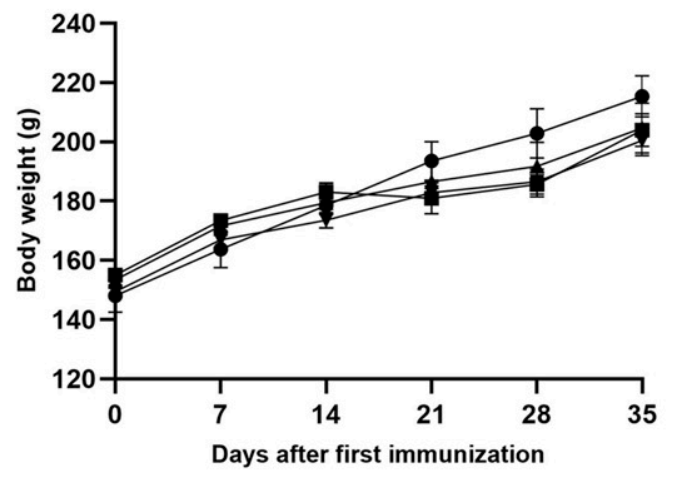

B

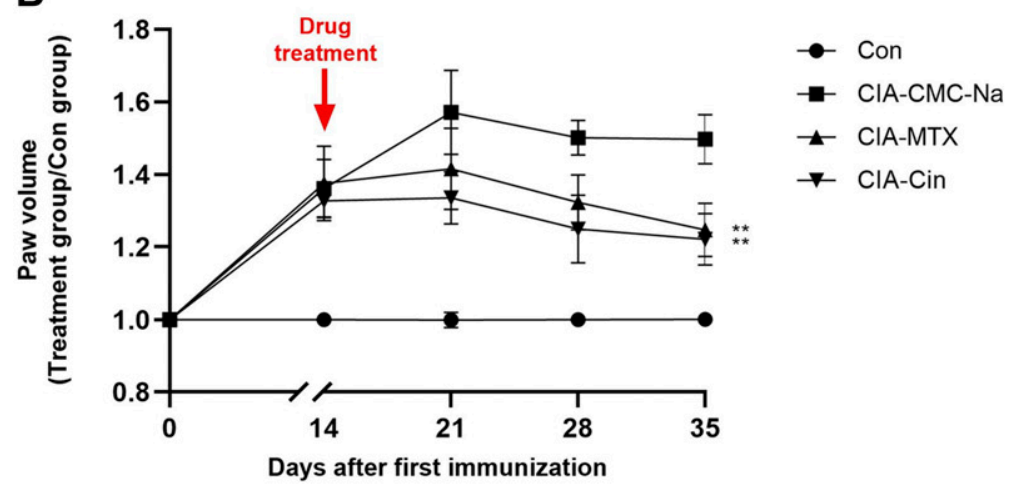

- Con

- CIA-MTX

$\rightarrow \quad$ CIA-Cin
D
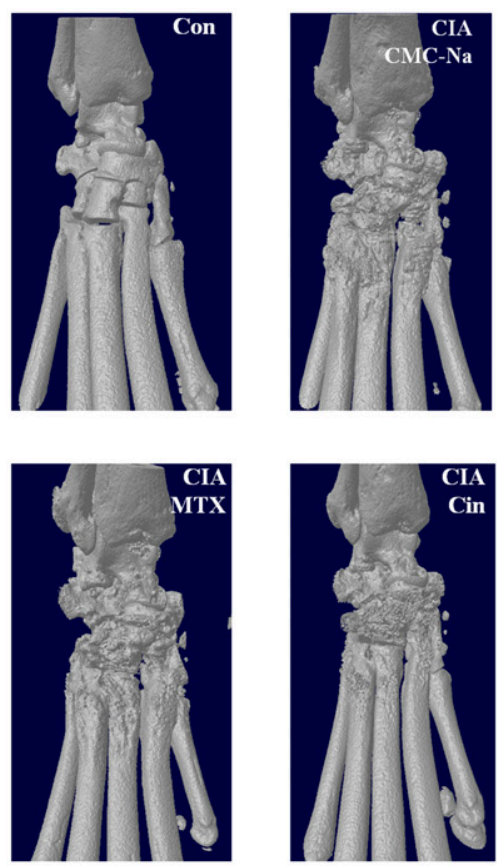

F

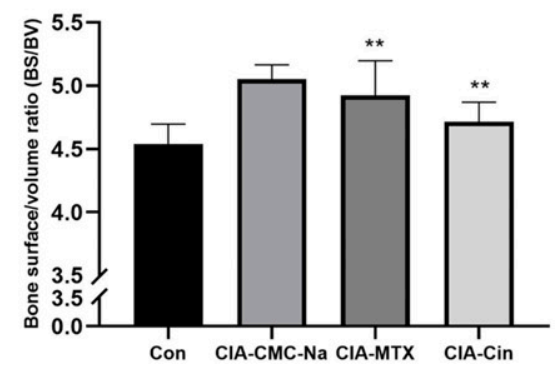

C

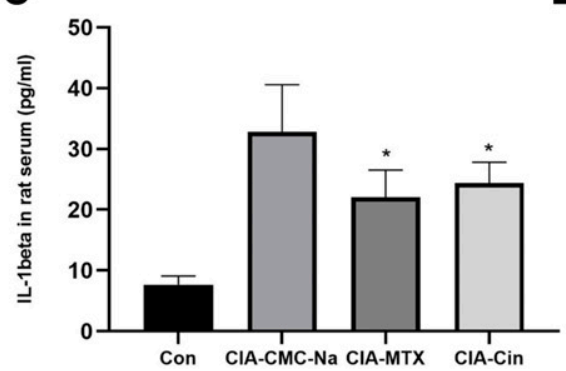

E

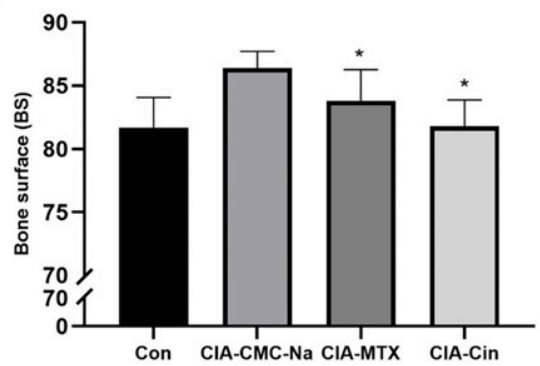

Fig. 5. Cin attenuated the symptoms of CIA rats and joint destruction. (A) The body weight. (B) Hind paw volume was recorded every 7 days after drug treatment. (C) The serum IL- $1 \beta$ levels decreased in the CIA-treated rats with Cin or MTX group. (D) On day 21 after being drug treated, rats were sacrificed. 3D reconstructions of the micro-CT analysis from CIA rats using the CTAn software. The bone radiologic destruction parameters BS (E) and BS:BV ratio $(\mathrm{F})$ were attributed from the micro-CT analysis. The ROI included the region between the metatarsal and tarsal bones. $N=6$. $* P<0.05$; $* * P<0.01$, the comparison was performed between treatment and vehicle control group.

that, with the IL- $1 \beta$ stimulation, JAK2 was promptly phosphorylated while p-STAT1 and p-STAT3 phosphorylated. The results indicated that in the IL- $1 \beta$-induced inflammatory cascade, JAK2, is at the upstream. Both Cin and Jak 2 inhibitor decreased the expression of p-STAT1 and p-STAT3 phosphorylation in human synoviocyte MH7A cells, whereas Cin also suppressed JAK2 phosphorylation, which hinted that Cin was effective on the upstream of JAK2. However, the direct target of Cin has not been found yet. In summary, Cin may become a new small-molecule inhibitor of JAK/STAT signaling to attenuate the inflammatory response.

The transcription factor NF- $\kappa$ B plays a crucial role in the inflammation processes of many tissues (Ray et al., 1995; Ju Hwang et al., 2019). Inhibition of $\mathrm{NF}-\kappa \mathrm{B}$ transcriptional activity is an important way for anti-inflammation in osteoarthritis and RA (Park et al., 2016; Jiang et al., 2017). The NF$\kappa \mathrm{B}$ protein dimer is complexed with the inhibitory proteins,
$\mathrm{I} \kappa \mathrm{Bs}(\mathrm{I} \kappa \mathrm{B} \alpha, \mathrm{I} \kappa \mathrm{B} \beta)$ in the inactivated state and is sequestered in the cytoplasm (Hong, 2017). After the stimulation of proinflammation factors, such as LPS, TNF- $\alpha$, or IL- $1 \beta$, the inhibitory proteins $\mathrm{I} \kappa \mathrm{B} \alpha$ is rapidly phosphorylated by $\mathrm{IKK} \alpha / \beta$, and ubiquitinated, rapidly degraded by the proteasome, allowing NF- $\kappa \mathrm{B}$ to release from $\mathrm{I} \kappa \mathrm{B}$ and translocate to the nucleus and initiate transcription by binding to numerous specific gene promoter elements to stimulate inflammation (Gallo et al., 2017). In our study, although these proinflammatory factors were all significantly increased after IL-1 $\beta$ stimulated, phosphorylation of an important transcription factor in the inflammation processes, NF- $\kappa \mathrm{B}$, was not changed obviously after IL-1 $\beta$ stimulation. Compared with our previous work (Jia et al., 2015), it is found that the cells used in this study were in an inflammation state originally. However, the decrease of these cytokines with statistical analysis suggests that Cin is effective for anti-inflammation in human synoviocytes-MH7A cells. 


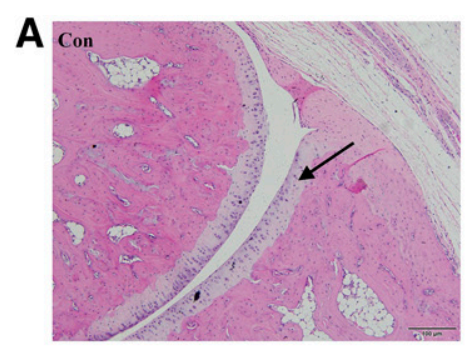

B

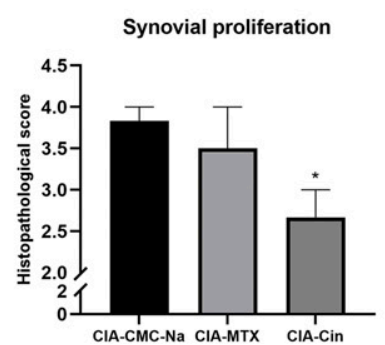

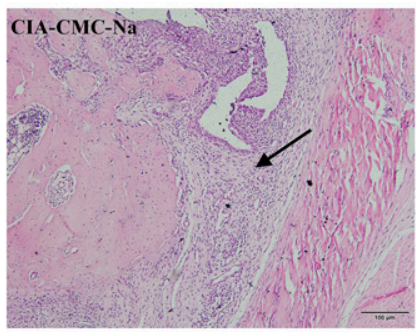

C

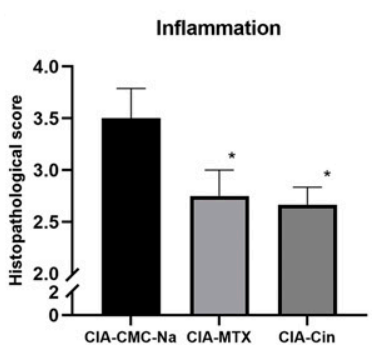

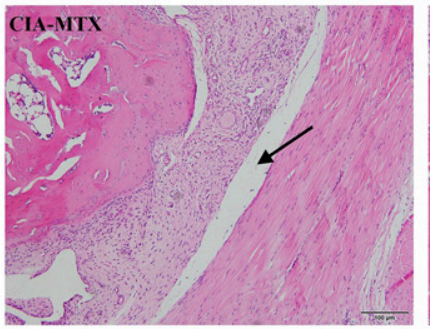

D

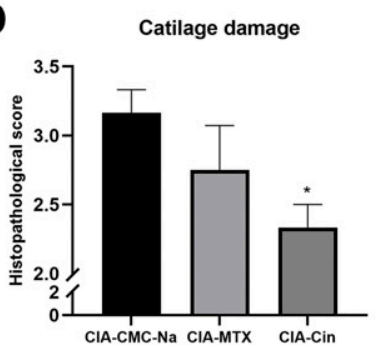

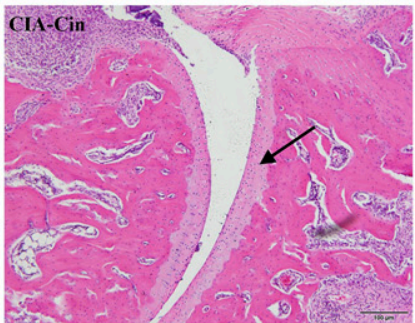

E

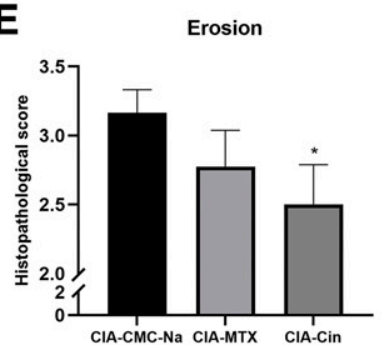

Fig. 6. The histopathological severity was assessed and calculated histologic findings. (A) The specimens with removed arthritic paws were stained with H\&E (original magnification, $100 \times$ ). The histopathological severity in terms of synovial proliferation (B), inflammation (C), cartilage damage (D), and bone surface erosion (E) were assessed and calculated. The ROI included the region between the metatarsal and tarsal bones. $N=6$. $* P<0.05 ; P<0.01$, the comparison was performed between treatment and vehicle control group.

Recently, numerous studies have shown that the Akt and ERK signaling pathways play essential roles in the pathogenesis of inflammation by phosphorylation of $\mathrm{I}_{\kappa} \mathrm{B}$ (Yang et al., 2016; Wang et al., 2017). It is reported that hemolysate increases the phosphorylation of Akt and ERK1/2 promotes the degradation of $\mathrm{I} \kappa \mathrm{B} \alpha$ and subsequently increases the nuclear translocation of NF- $\kappa \mathrm{B}$ (Yang et al., 2016). Thus, Akt and ERK signaling pathways could be the main upstream of NF- $\kappa$ B signaling cascades (Dan et al., 2008; Sun et al., 2017). Our results showed that Cin could increase the phosphorylation of Akt at Thr308 but decrease the phosphorylation of NF$\kappa \mathrm{B}$, which explained the slight effect of Cin on suppression of $\mathrm{NF}-\kappa \mathrm{B}$ phosphorylation and hinted that there were other regulators at the upstream of $\mathrm{NF}-\kappa \mathrm{B}$ in IL- $1 \beta$-induced human synoviocyte MH7A cells.

The most important goal in RA therapy is to maintain normal joint function via prevention of bone destruction ( $\mathrm{Li}$ et al., 2017; Tanaka, 2019). In the present study, we investigated Cin could attenuate the severity of CIA, in addition to suppressing inflammatory disease activity and preventing bone destruction. Blocking of IL-1 is a cartilage- and boneprotective therapy in destructive arthritis. Activated RA-FLSs are the major source of proinflammatory cytokines and inflammatory mediators in the synovial tissue. Therefore, the inhibition of inflammatory cytokines can significantly reduce RA-FLSs cartilage invasiveness (Joosten et al., 1999; Ganesan and Rasool, 2017). Our results showed that Cin markedly attenuated IL-1 $\beta$ level and arthritis symptom in CIA rats. Meanwhile, bone erosion in the RA control group was markedly more extensive than that in the Cin treatment group. Combined with the results of in vitro study, Cin might prevent bone destruction via inhibited fibroblast synovial cells producing IL- $1 \beta$.

Our findings indicated that Cin significantly inhibited release of the proinflammatory cytokine both the rats with CIA and human synoviocyte MH7A cell, partly modulating through the JAK/STAT pathway. Importantly, Cin markedly ameliorated arthritis in rats and conferred joint protection when used therapeutically. Taken together, these findings suggest that Cin may be a potential therapeutic agent for chronic inflammatory disorders such as RA.

\section{Acknowledgments}

We thank Dr. Qing-Wen Wang (Peking University Shenzhen Hospital) for providing primary synovial cells from the rheumatoid arthritis patients.

\section{Authorship Contributions}

Participated in research design: Qin, X.-L. Wang.

Conducted experiments: Cheng, Zhong, Meng, Zheng.

Performed data analysis: Cheng, Zhong, Meng, Zhang, Y. Wang, X.-L. Wang.

Wrote or contributed to the writing of the manuscript: Cheng, Zhong, Qin, X.-L. Wang.

\section{References}

Adach A, Ellert-Miklaszewska A, and Kaminska B (2009) Molecular characterization of STAT signaling in inflammation and tumorigenesis. Methods Mol Biol 512: $265-278$.

Ahmad SF, Ansari MA, Zoheir KM, Bakheet SA, Korashy HM, Nadeem A, Ashour $\mathrm{AE}$, and Attia SM (2015) Regulation of TNF- $\alpha$ and NF- $\mathrm{KB}$ activation through the JAK/STAT signaling pathway downstream of histamine 4 receptor in a rat model of LPS-induced joint inflammation. Immunobiology 220:889-898.

Antill AC, Ballard DH, Hollister AM, Rogers EJ, Yang S, and Lokitz SJ (2016) MicroCT evaluation of rheumatoid arthritis mouse model disease progression: manual tracings versus semi-automated routines. Diagn Interv Imaging 97:651-655.

Brand DD, Latham KA, and Rosloniec EF (2007) Collagen-induced arthritis. Nat Protoc 2:1269-1275.

Cassuto J, Folestad A, Göthlin J, Malchau H, and Kärrholm J (2018) The key role of proinflammatory cytokines, matrix proteins, RANKL/OPG and Wnt/3-catenin in bone healing of hip arthroplasty patients. Bone 107:66-77.

Castejón ML, Rosillo MA, Montoya T, González-Benjumea A, Fernández-Bolaños JG, and Alarcón-de-la-Lastra C (2017) Oleuropein down-regulated IL-1 $\beta$-induced inflammation and oxidative stress in human synovial fibroblast cell line SW982 [published correction appears in Food Funct (2017) 8:2341]. Food Funct 8: 1890-1898.

Chao LK, Hua KF, Hsu HY, Cheng SS, Lin IF, Chen CJ, Chen ST, and Chang ST (2008) Cinnamaldehyde inhibits pro-inflammatory cytokines secretion from monocytes/macrophages through suppression of intracellular signaling. Food Chem Toxicol 46:220-231.

Chow DH, Zheng L, Tian L, Ho KS, Qin L, and Guo X (2018) Application of ultrasound accelerates the decalcification process of bone matrix without affecting histological and immunohistochemical analysis. J Orthop Translat 17:112-120.

Dan HC, Cooper MJ, Cogswell PC, Duncan JA, Ting JP, and Baldwin AS (2008) Aktdependent regulation of NF-kappaB is controlled by mTOR and Raptor in association with IKK. Genes Dev 22:1490-1500. 
Feng Z, Zheng W, Li X, Lin J, Xie C, Li H, Cheng L, Wu A, and Ni W (2017) Cryptotanshinone protects against IL-1ß-induced inflammation in human osteoarthritis chondrocytes and ameliorates the progression of osteoarthritis in mice. Int Immunopharmacol 50:161-167.

Gallo J, Raska M, Kriegova E, and Goodman SB (2017) Inflammation and its resolution and the musculoskeletal system. J Orthop Translat 10:52-67.

Ganesan R and Rasool M (2017) Fibroblast-like synoviocytes-dependent effector molecules as a critical mediator for rheumatoid arthritis: current status and future directions. Int Rev Immunol 36:20-30.

Geyer M and Schonfeld C (2018) Novel insights into the pathogenesis of osteoarthritis. Curr Rheumatol Rev 14:98-107.

Gibon E, Lu LY, Nathan K, and Goodman SB (2017) Inflammation, ageing, and bone regeneration. J Orthop Translat 10:28-35.

Gunawardena D, Karunaweera N, Lee S, van Der Kooy F, Harman DG, Raju R Bennett L, Gyengesi E, Sucher NJ, and Münch G (2015) Anti-inflammatory activity of cinnamon (C. zeylanicum and C. cassia) extracts - identification of E-cinnamaldehyde and o-methoxy cinnamaldehyde as the most potent bioactive compounds. Food Funct 6:910-919.

Guo C, Yang L, Luo J, Zhang C, Xia Y, Ma T, and Kong L (2016) Sophoraflavanone G from Sophora alopecuroides inhibits lipopolysaccharide-induced inflammation in RAW264.7 cells by targeting PI3K/Akt, JAK/STAT and Nrf2/HO-1 pathways. Int Immunopharmacol 38:349-356.

Hayer S, Bauer G, Willburger M, Sinn K, Alasti F, Plasenzotti R, Shvets T, Niederreiter B, Aschauer C, Steiner G, et al. (2016) Cartilage damage and bone erosion are more prominent determinants of functional impairment in longstanding experimental arthritis than synovial inflammation. Dis Model Mech $\mathbf{9}$ 1329-1338.

Ju Hwang C, Choi DY, Park HM, and Hong JT (2019) NF-кB as a key mediator of brain inflammation in Alzheimer's disease. CNS Neurol Disord Drug Targets 18: $3-10$

Huang B, Yuan HD, Kim DY, Quan HY, and Chung SH (2011) Cinnamaldehyde prevents adipocyte differentiation and adipogenesis via regulation of peroxisome proliferator-activated receptor- $\gamma(\mathrm{PPAR} \gamma)$ and AMP-activated protein kinase (AMPK) pathways. $J$ Agric Food Chem 59:3666-3673.

Huang JS, Lee YH, Chuang LY, Guh JY, and Hwang JY (2015) Cinnamaldehyde and nitric oxide attenuate advanced glycation end products-induced the Jak/STAT signaling in human renal tubular cells. J Cell Biochem 116:1028-1038.

Jia Q, Cheng W, Yue Y, Hu Y, Zhang J, Pan X, Xu Z, and Zhang P (2015) Cucurbitacin $\mathrm{E}$ inhibits TNF- $\alpha$-induced inflammatory cytokine production in human synoviocyte MH7A cells via suppression of PI3K/Akt/NF-кB pathways. Int Immunopharmacol 29:884-890.

Jiang Y, Zhu L, Zhang T, Lu H, Wang C, Xue B, Xu X, Liu Y, Cai Z, Sang W, et al (2017) BRD4 has dual effects on the HMGB1 and NF-kB signalling pathways and is a potential therapeutic target for osteoarthritis. Biochim Biophys Acta Mol Basis Dis 1863:3001-3015.

Joosten LA, Helsen MM, Saxne T, van De Loo FA, Heinegard D, and van Den Berg WB (1999) IL-1 alpha beta blockade prevents cartilage and bone destruction in murine type II collagen-induced arthritis, whereas TNF-alpha blockade only ameliorates joint inflammation. J Immunol 163:5049-5055.

Kang JD, Georgescu HI, McIntyre-Larkin L, Stefanovic-Racic M, and Evans CH (1995) Herniated cervical intervertebral discs spontaneously produce matrix metalloproteinases, nitric oxide, interleukin-6, and prostaglandin E2. Spine 20 2373-2378.

Kim BH, Lee YG, Lee J, Lee JY, and Cho JY (2010) Regulatory effect of cinnamaldehyde on monocyte/macrophage-mediated inflammatory responses. Mediators Inflamm 2010:529359.

Kim DH, Kim CH, Kim MS, Kim JY, Jung KJ, Chung JH, An WG, Lee JW, Yu BP, and Chung HY (2007) Suppression of age-related inflammatory NF-kappaB activation by cinnamaldehyde. Biogerontology 8:545-554

Li J, Teng Y, Liu S, Wang Z, Chen Y, Zhang Y, Xi S, Xu S, Wang R, and Zou X (2016) Cinnamaldehyde affects the biological behavior of human colorectal cancer cells and induces apoptosis via inhibition of the PI3K/Akt signaling pathway. Oncol Rep 35:1501-1510.

Li N, Xu Q, Liu Q, Pan D, Jiang Y, Liu M, Liu M, Xu H, and Lin C (2017) Leonurine attenuates fibroblast-like synoviocyte-mediated synovial inflammation and joint destruction in rheumatoid arthritis. Rheumatology (Oxford) 56:1417-1427.

Liao JC, Deng JS, Chiu CS, Hou WC, Huang SS, Shie PH, and Huang GJ (2012) Antiinflammatory activities of Cinnamomum cassia constituents in vitro and in vivo. Evid Based Complement Alternat Med 2012:429320.

Lim H and Kim HP (2011) Matrix metalloproteinase-13 expression in IL-1 $\beta$-treated chondrocytes by activation of the p38 MAPK/c-Fos/AP-1 and JAK/STAT pathways. Arch Pharm Res 34:109-117.

Park SY, Lee SW, Kim HY, Lee SY, Lee WS, Hong KW, and Kim CD (2016) SIRT1 inhibits differentiation of monocytes to macrophages: amelioration of synovial inflammation in rheumatoid arthritis. J Mol Med (Berl) 94:921-931.

Qi QR and Yang ZM (2014) Regulation and function of signal transducer and activator of transcription 3. World J Biol Chem 5:231-239.

Rahmati M, Mobasheri A, and Mozafari M (2016) Inflammatory mediators in osteoarthritis: a critical review of the state-of-the-art, current prospects, and future challenges. Bone 85:81-90.

Ray A, Siegel MD, Prefontaine KE, and Ray P (1995) Anti-inflammation: direct physical association and functional antagonism between transcription factor NF$\mathrm{KB}$ and the glucocorticoid receptor. Chest 107 (3 Suppl):139S.

Sluzalska KD, Liebisch G, Lochnit G, Ishaque B, Hackstein H, Schmitz G, Rickert M, and Steinmeyer J (2017) Interleukin-1 $\beta$ affects the phospholipid biosynthesis of fibroblast-like synoviocytes from human osteoarthritic knee joints. Osteoarthritis Cartilage 25:1890-1899.

Sun H, Zhu X, Cai W, and Qiu L (2017) Hypaphorine attenuates lipopolysaccharideinduced endothelial inflammation via regulation of TLR4 and PPAR- $\gamma$ dependent on PI3K/Akt/mTOR signal pathway. Int J Mol Sci 18 (44).

Swärd P, Frobell R, Englund M, Roos H, and Struglics A (2012) Cartilage and bone markers and inflammatory cytokines are increased in synovial fluid in the acute phase of knee injury (hemarthrosis)--a cross-sectional analysis. Osteoarthritis Cartilage 20:1302-1308.

Tanaka Y (2019) Clinical immunity in bone and joints. J Bone Miner Metab 37:2-8. Tsuji-Naito K (2008) Aldehydic components of cinnamon bark extract suppresses RANKL-induced osteoclastogenesis through NFATc1 downregulation. Bioorg Med Chem 16:9176-9183.

Wang X, Yang L, Yang L, Xing F, Yang H, Qin L, Lan Y, Wu H, Zhang B, Shi H, et al. (2017) Gypenoside IX suppresses p38 MAPK/Akt/NFkB signaling pathway activation and inflammatory responses in astrocytes stimulated by proinflammatory mediators. Inflammation 40:2137-2150.

Weng SJ, Yan DY, Tang JH, Shen ZJ, Wu ZY, Xie ZJ, Yang JY, Bai BL, Chen L, Boodhun V, et al. (2019) Combined treatment with Cinnamaldehyde and $\beta$-TCP had an additive effect on bone formation and angiogenesis in critical size calvarial defect in ovariectomized rats. Biomed Pharmacother 109:573-581.

Yang XL, Kim CK, Kim TJ, Sun J, Rim D, Kim YJ, Ko SB, Jang H, and Yoon BW (2016) Anti-inflammatory effects of fimasartan via Akt, ERK, and NFкB pathways on astrocytes stimulated by hemolysate. Inflamm Res 65:115-123.

Yuan Y, Chen Y, Zhou Z, Jiao Y, Li C, Zheng Y, Lin Y, Xiao J, Chen Z, and Cao P (2018) Association between chronic inflammation and latent infection of Propionibacterium acnes in non-pyogenic degenerated intervertebral discs: a pilot study. Eur Spine J 27:2506-2517.

Zheng LZ, Wang JL, Kong L, Huang L, Tian L, Pang QQ, Wang XL, and Qin L (2018) Steroid-associated osteonecrosis animal model in rats. J Orthop Translat 13:13-24.

Address correspondence to: Dr. Xin-Luan Wang, Translational Medicine R\&D Center, Institute of Biomedical and Health Engineering, Shenzhen Institutes of Advanced Technology, 1068 Xueyuan Ave, Shenzhen University Town, Shenzhen 518055, P.R. China. E-mail: xl.wang@siat.ac.cn 